\title{
Dealing with Front-End White Noise on Differentiated Measurements such as Frequency and ROCOF in Power Systems
}

\author{
Andrew J. Roscoe, Roscoe, Senior Member, IEEE, \\ Steven M. Blair, Member, IEEE \\ Department of Electronic and Electrical Engineering \\ University of Strathclyde \\ Glasgow, UK
}

\author{
William Dickerson, Fellow, IEEE \\ Arbiter Systems, Paso Robles, California, USA \\ Gert Rietveld, Senior Member, IEEE \\ VSL, Delft, The Netherlands.
}

This paper describes the way that white noise (including quantised input section sampling) imparts errors onto frequency and rate-of-change-of-frequency (ROCOF) measurements. The main paper focus concerns the use of filtered heterodyned (i.e. Fourier) analyses for single-phase and 3-phase systems, and the filtered Clarke transform for 3-phase systems. The rules and equations governing the effect of white noise on frequency and ROCOF are formulated for these techniques, explaining the subtle effects of aliasing, splitting signals and noise into their positive and negative frequency components, and the correlation or de-correlation of noise. It is shown that - as expected - for 3phase AC measurements, averaging 3 single-phase Fourier measurements produces the same performance against noise as using a method based on Clarke's transform, if identical filtering is used. Furthermore, by understanding the theory behind the frequency and ROCOF measurement processes, it is shown that to achieve the lowest RMS errors, in the presence of front-end white noise (alone, ignoring other dynamic signal and power quality aspects), a filter which provides $\sim 40 \mathrm{~dB} /$ decade attenuation (i.e. a 2-boxcar cascade) is recommended for a frequency measurement, but a filter which rolls off at $\sim \mathbf{6 0}$ $\mathrm{dB} /$ decade (i.e. a 3-boxcar cascade) is recommended for a ROCOF measurement.

Keywords-Frequency measurement, Frequency estimation, White noise, Gaussian noise, Colored noise, Signal to noise ratio, Power system measurements, Finite impulse response filters, Fourier transforms, Phasor Measurement Units.

\section{INTRODUCTION.}

Frequency has always been, and will continue to be, a key parameter within AC power systems. Measurement of frequency is required both within frequency and active-power control loops, to despatch generators, storage and/or loads, and also within protective devices to monitor over-or-underfrequency events. ROCOF (Rate of Change of Frequency) has been historically important as a measurand for protective

This work is part of the EU joint research project "Standard tests and requirements for rate-of-change of frequency (ROCOF) measurements in smart grids" which has received funding from the EMPIR programme co-financed by the Participating States and from the European Union's Horizon 2020 research and innovation programme. devices which attempt to determine unintentional islanding events. However, the measurand ROCOF is now finding many new applications within power systems containing high penetrations of renewables and other converter-connected devices such as DC links, storage devices, electric vehicles, and industrial loads. For example, the GridMetrix [1] system attempts to estimate system inertia using a network of distributed phasor measurement units (PMUs) which need to make accurate ROCOF measurements.

While the amplitude and phase of an AC signal are relatively easy to determine, to usable accuracies, using short time windows such as 1 or 2 cycles, the determination of accurate frequency and ROCOF is much more problematic. Noise and imperfections of the AC power quality (particularly interharmonics) can have a dramatic effect on measurement accuracy [2]. This is because frequency is determined from phase via a differential against time. The transfer function of differentiation has a gain that linearly increases with frequency, amplifying the effects of unwanted wideband noise and other interferences that occur at frequencies outside the passband. ROCOF is calculated via an additional differentiation from frequency, further exacerbating the problems.

This paper focusses on the effects of front-end white noise, introduced by analogue instrumentation, and ADC (Analogue to Digital Converter) quantisation effects. These introduce white or "nearly white" noise into the digital measurement process. They can result in an excessively noisy frequency measurement, and a ROCOF measurement in which the actual ROCOF magnitude is often swamped by noise, with measurand SNR $<0 \mathrm{~dB}$ [1]. Previously, [3] analysed this issue, but only considered rectangular filter windows and consequently made very pessimistic estimates of performance. The analysis was extended to include other windows in [4], but the effect of windowing was determined by time-domain fullalgorithm Monte-Carlo simulation, rather than analytically. Frequency-domain methods to analytically consider filter/window design were briefly presented in [5].

This paper builds on [5], presenting a more rigorous description of the practical effect of noise. The paper provides 
new formulations of the errors; formulations which are the analytical tools required to quickly (without simulation) estimate the resulting noise on frequency and ROCOF measurement, for given sampling and measurement processes, and for any filter/window design. This de-risks the selection of important device parameters like sample rate, analogue SNR (signal-to-noise ratio), ADC resolution, aperture jitter, input scaling, and filter design. The understanding gained through the development of these tools also has an additional benefit; it is now possible to suggest "optimal" filters which provide the best performance against white noise.

This paper concentrates on the use of two common techniques used within 3-phase AC power systems: filtered heterodyned phase-by-phase (DFT, Discrete Fourier Transform) analyses, and the filtered Clarke transform. The rules and equations governing the effect of white noise on frequency and ROCOF are formulated for these techniques, explaining the subtle effects of aliasing, splitting signals (and noise) into their positive and negative frequency components, and the correlation or de-correlation of noise. Zero-crossing and phased-locked-loop techniques are also considered for comparison.

\section{THE HETERODYNE PROCESS}

The classic way to measure a single-phase AC power system fundamental carrier component is to use a heterodyne process followed by a filtering stage. The combination of these two components forms a Discrete Fourier Transform (DFT) which reveals the amplitude and phase of the input waveform, at the tuned frequency $f_{T}$ of the heterodyne quadrature oscillator. An overview of the process is show in Fig. 1.

In Fig. 1, a single real signal enters at point $\mathrm{A}$. The real signal hopefully has a dominant fundamental sinusoidal carrier at a positive frequency $f_{\mathrm{C}}$. It will also contain white noise due to analogue components and ADC quantisation, and other signals at harmonic or inter-harmonic frequencies. Dealing with these other components is outside the main scope of this paper, but it is discussed in [6]. In the context of this paper, the components of primary interest are the carrier at frequency $f_{C}$ and noise components at frequencies $f_{N}$. To understand and quantify the interaction of these components at point $\mathrm{E}$, the impact on the measurement $\mathbf{V}$, it is necessary to examine very carefully the entire process through points A, B, C, D and E.

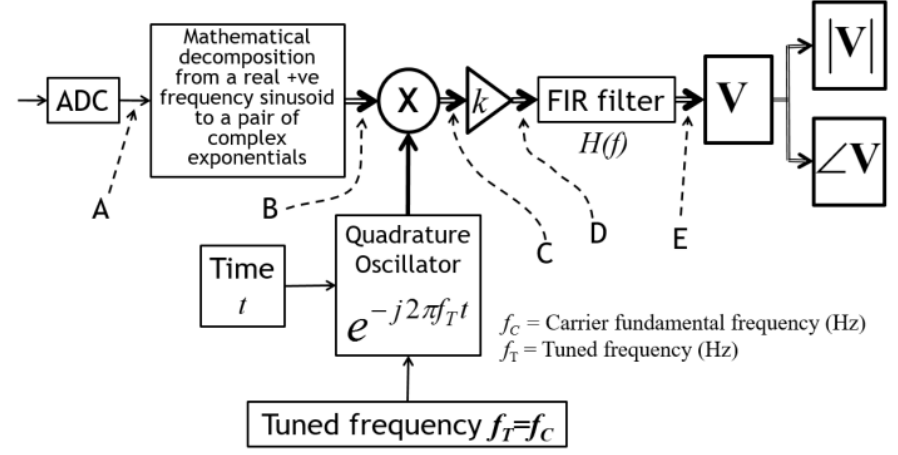

Consider a single positive-frequency real-valued signal component entering at point A of Fig. 1, with an RMS magnitude of $A_{R M S}$.

$$
V_{A}(t)=A_{R M S} \cdot \sqrt{2} \cos (2 \pi f t)
$$

Between point $\mathrm{A}$ and point $\mathrm{B}$ of Fig. 1, a mathematical decomposition of this real signal effectively occurs. This is not a physical splitting, merely a different mathematical way of considering the signal, split into its positive and negative frequency components, as a pair of complex exponentials. It is easy to become confused at this point, thinking that the signal has been split into two parts, each with half the amplitude and one quarter of the original power. But, this is not true. The two split signals can still be reconsidered to re-combine back into the form of (1), interfering constructively since they are entirely correlated.

$$
\mathbf{V}_{\mathbf{B}}(t)=\left(\frac{A_{R M S}}{2}\right)\left(e^{j 2 \pi f t}+e^{-j 2 \pi f t}\right) \sqrt{2}
$$

Between points B and C, a quadrature oscillator is applied, which is tuned to a frequency $f_{T}$. Therefore, at point $\mathrm{C}$ of Fig. 1:

$$
\begin{aligned}
& \mathbf{V}_{\mathbf{C}}(t)=\left(\frac{A_{R M S}}{2}\right) e^{-j 2 \pi f_{T} t}\left(e^{j 2 \pi f t}+e^{-j 2 \pi f t}\right) \sqrt{2} \\
& \mathbf{V}_{\mathbf{C}}(t)=\left(\frac{A_{R M S}}{2}\right)\left(e^{j 2 \pi\left(f-f_{T}\right) t}+e^{j 2 \pi\left(-f-f_{T}\right) t}\right) \sqrt{2}
\end{aligned}
$$

In a well-designed application, frequency-tracking is employed, and $f_{T}$ is set equal to the wanted carrier fundamental frequency $f_{C}$.

$$
f_{T}=f_{C}
$$

This results in one of the complex exponentials due to the carrier falling at $\left(f_{C}-f_{T}\right)=0 \mathrm{~Hz}$, i.e. having a steady-state phase, and the other one (called the image) having a frequency of $\left(-f_{C}-f_{T}\right)=-2 f_{C}=-2 f_{T}$. This will be filtered out later by $H(f)$, which is normally designed to place deep notches at $\pm 2 f_{T}$.

$$
\mathbf{V}_{\mathbf{C}_{-} \text {Carrier }}(t)=\left(\frac{A_{R M S}}{2}\right)\left(1+e^{j 2 \pi\left(-2 f_{C}\right) t}\right) \sqrt{2}
$$

The magnitudes of the complex exponentials are not changed between points B and C. Only their frequencies are shifted downwards by $f_{T}$. Also, a key point of understanding is that the two signal parts of each pair become de-correlated, and cannot be recombined into the form (1). If one of the carrier components is later removed through filtering, it does represent a genuine halving of the carrier amplitude, with apparent loss of $3 / 4$ of the power: a signal loss of $6 \mathrm{~dB}$.

Between point $C$ and point D of Fig. 1, a gain of $k=\sqrt{ } 2$ is shown. Other values could be used, with no changes to the final results of this paper, since both carrier and noise will be affected equally. However, $k=\sqrt{2}$ is the most convenient choice since it produces phasors at point $\mathrm{D}$ with magnitudes

Fig. 1 The heterodyning and filtering process 
that are equal to the RMS amplitudes of the corresponding real input signals at point $\mathrm{A}$ :

$$
\mathbf{V}_{\mathbf{D}}(t)=A_{R M S}\left(e^{j 2 \pi\left(f-f_{T}\right) t}+e^{j 2 \pi\left(-f-f_{T}\right) t}\right)
$$

The final stage of the process, between points $\mathrm{D}$ and $\mathrm{E}$ of Fig. 1, is a filter, with frequency response $H(f)$. This filter has unity gain at $0 \mathrm{~Hz}$, but is designed to reject the image component at $-2 f_{T}$, and also to reject wideband noise and any other unwanted out-of-band interference components. $H(f)$ could be an IIR (Infinite Impulse Response) or a FIR (Finite Impulse Response) filter. However, FIR filters are particularly useful since deep notches can often conveniently be placed at the image frequency, and linear phase response can be achieved. In particular, the use of a rectangular (boxcar) filter as the Dirichlet kernel results in Fig. 1 becoming a conventional rectangular-windowed DFT. However, much more complex windows and filters can be applied, with responses and latencies tailored to a particular application. In this paper, FIR filters are created by cascading tunable boxcar filters together to provide effective image and harmonic rejection, even while frequency varies from nominal. Some of the concepts were laid down in [7], although the method we use is unconditionally (not marginally) stable, allows the use of windows that are non-integer numbers of samples, and allows the use of floating-point arithmetic without overflow or loss of precision. The method used is described in [8-10] and operates extremely quickly in real-time [11]. To include the adaptive tuning of $f_{T}$ to the measured value of $f_{C}$ in Fig. 1 also requires a careful breaking of the feedback loop so that the system does not inherit a resonant IIR property like a PLL (Phased Locked Loop). This is achieved using the "TickTock" method described in [12]

The heterodyne process between points $\mathrm{A}$ and $\mathrm{C}$ of Fig. 1 can be explained visually using Fig. 2 to Fig. 4, which show a frequency-domain representation of the signal-splitting and heterodyning process when there are two discrete signal components present in the real-valued sampled signal: a carrier fundamental at $+f_{C}$, and a single noise or interference component at $+f_{N}$. The heterodyne oscillator is set so that $f_{T}=f_{C}$.

Fig. 2 shows the two real-valued signals with positive frequencies $+f_{C}$ and $+f_{N}$. Fig. 3 shows the signals split into two pairs of complex exponentials with both positive and negative frequencies, and halved amplitudes.

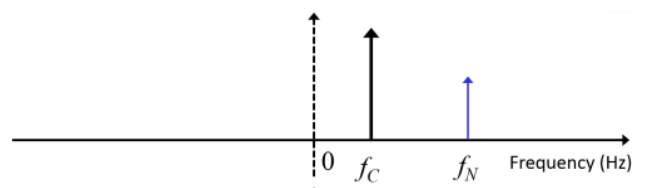

Fig. 2 A carrier at frequency $f_{C}\left(=f_{T}\right)$ and noise at $f_{N}$ : signal considered as real, with only positive frequency components, at point A of Fig. 1.

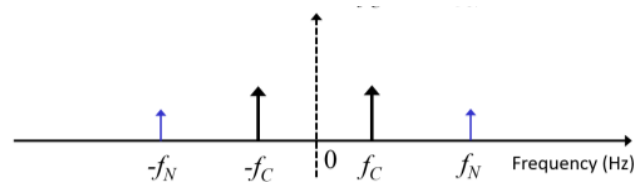

Fig. 3 A carrier at frequency $f_{C}\left(=f_{T}\right)$ and noise at $f_{N}$ : signals split into complex positive and negative frequency components with halved amplitudes at point B of Fig. 1 .

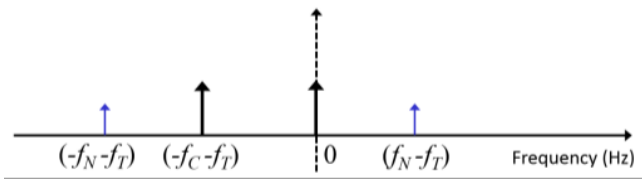

Fig. 4 Heterodyning of a carrier at frequency $f_{C}\left(=f_{T}\right)$ and noise at $f_{N}$ : heterodyning applied to shift all complex components down in frequency by $f_{T}$, at point $\mathrm{C}$ of Fig. 1.

Fig. 4 shows the total expected content of the complex signal $\mathbf{V}_{\mathbf{C}}$ in this scenario. The wanted measurement result contains only the component at $0 \mathrm{~Hz}$, which represents a steady-state phasor measurement of the carrier. The function of filtering is to remove the unwanted components, in particular the image at $\left(-f_{C}-f_{T}\right)$ which has the same amplitude as the component at $0 \mathrm{~Hz}$, and also, most relevantly for this paper, at all frequencies other than $0 \mathrm{~Hz}$, where noise components can and will fall.

\section{NOISE SOURCES AND SPECTRAL DENSITY}

When a signal is sampled with an ADC, the digitised signal contains the wanted signal, plus noise which arises via four mechanisms. The noise degrades the Signal to Noise Ratio (SNR) from infinity, to some finite number expressed in $\mathrm{dB}$.

Firstly, all analogue input circuitry introduces a level of unavoidable white noise. Second, some applications apply ADC dithering techniques to improve linearity, which deliberately add white noise to the analogue signal. Third, the signal is quantised as it is sampled by the ADC, and assigned a digital value. The ADC quantisation is (in theory) perfect, but in reality also exhibits some level of INL (Integral NonLinearity) and DNL (Differential Non-Linearity). The fourth consideration is the ADC clock aperture jitter. Its effect can be estimated by [13]:

$$
S N R_{d B \text { Jitter }}=-20 \log \left(2 \pi f_{C} \cdot t_{R M S}\right)
$$

where $t_{R M S}$ is the ADC clock aperture jitter (which should be much smaller than, and distinguished from, clock accuracy \& wander). In most commercial power system applications, noise due to jitter is acceptably low, since $f_{C}$ is low $(50-60 \mathrm{~Hz})$, and typically $t_{R M S}<1 \mathrm{~ns}$. For example the AD7863 ADC has an aperture jitter of 50ps [14], equating to a SNR of $\sim 156 \mathrm{~dB}$ which is high, so that its effect is insignificant to other noise mechanisms. However, clock jitters at 30ns or above [15] may have a noticeable impact on system performance.

The combined effect of all four mechanisms need to be considered, to estimate overall SNR for the sampling front end. Then, the following equation can be used to translate between SNR and ENOB (the Effective Number of Bits) [5, 14, 16, 17]: 
$S N R_{d B}=6.02 \cdot E N O B+1.76 \mathrm{~dB}$

A sampling process with $N$ bits will always have ENOB $\leq N$, by a quantity dependent on the analogue noise, and ADC imperfections. Some ADCs provide a pre-calculated estimate of inherent ENOB (e.g. [14]), which account for several of the mechanisms, at least in part. However, the ENOB may be further degraded in the final application, due to analogue sensors, cables, circuits or amplifiers in the signal chain.

By convention, quoted ENOB values assume that the measurement signal spans the full ADC range. However, in a practical application this is often not the case. ADCs need to be scaled so that they can capture signal amplitudes larger than nominal without clipping. At the same time, the most challenging measurement conditions include those where the signal amplitude is smaller than nominal. In these cases, the practical SNR is degraded, by:

$$
S N R_{\text {practical_dB }}=S N R_{d B}-20 \log _{10}\left(\frac{A_{\text {Fullscale }}}{A_{\text {Actual }}}\right) \mathrm{dB}
$$

Where $A_{\text {Fullscale }}$ is the maximum amplitude which can be measured without clipping, and $A_{\text {Actual }}$ is the actual signal amplitude.

For example, if a 16-bit ADC has an ENOB of 15, due to all the first four mechanisms, and the ADC is configured to allow a signal at $125 \%$ of nominal amplitude to be sampled without clipping, but the actual signal is at $80 \%$ of nominal amplitude, then the practical SNR will be:

$$
S N R_{\text {practical } \_d B}=6.02 \times 15+1.76-20 \log _{10}\left(\frac{1.25}{0.8}\right) \quad \mathrm{dB}
$$

which is a SNR of $88.2 \mathrm{~dB}$. This example value is used for investigations in section XI.

In a scenario with a known (or estimated) SNR, at a sampling frequency $f_{S}$, the relative power spectral density of noise $L_{d B c}(f)$ can be evaluated as $[5,16,17]$ :

$$
L_{d B c}(f)=-S N R_{d B}-10 \log _{10}\left(\frac{f_{S}}{2}\right) \mathrm{dBc} / \mathrm{Hz}
$$

$L_{d B c}(f)$ describes the relative level of noise compared to the carrier, and quantifies a constant white-noise density, across the whole positive-frequency interval between 0 and $f_{S} / 2$ (Nyquist). $L_{d B c}(f)$ can be used to derive $L(f)$, a linear noise power density, relative to a carrier with power 1 , with units of 1/Hz, by:

$$
L(f)=10^{\left(\frac{L_{d B c}(f)}{10}\right)} \quad 1 / \mathrm{Hz}
$$

Furthermore, the RMS noise amplitude density (relative to a carrier with unity RMS amplitude) can be expressed as:

RMS Noise Amplitude Density $=\sqrt{L(f)} \quad \sqrt{1 / H z}$
This means that if the RMS carrier amplitude is 1 at the ADC output, then the RMS noise amplitude density is $\sqrt{L(f)}$ across the frequency range $0<f<f_{S} / 2$.

\section{A. The effect of white noise between points $A$ and $C$}

Fig. 5 shows the rectangular shape of the noise amplitude density with units of $\sqrt{ }(1 / \mathrm{Hz})$. Every individual noise frequency component in Fig. 5 can be considered in the same way as the component $f_{N}$ in Fig. 2, and how it maps onto Fig. 3. As each noise component in Fig. 2 is a real positive-frequency sine wave (1) it can be regarded as being composed of two equal exponential components at positive and negative frequencies $+f_{N}$ and $-f_{N}$, each exponential component reduced in amplitude by half (2). This is shown in Fig. 6. The relative noise amplitude density at each frequency over $-f_{S} / 2<f<+f_{S} / 2$ is $\sqrt{L(f)} / 2$. But, even though it is noise, for every $f_{N}$, the pair of signals at $\pm f_{N}$ are still correlated with each other at point $\mathrm{B}$.

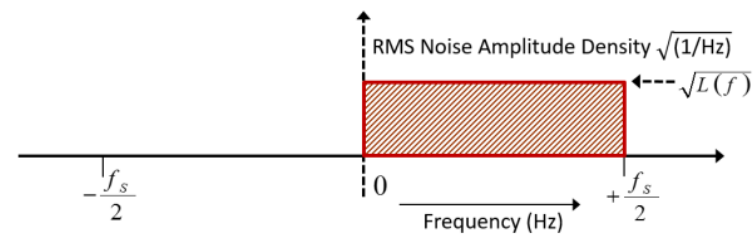

Fig. 5 Noise considered as real, with positive frequency only, at point A
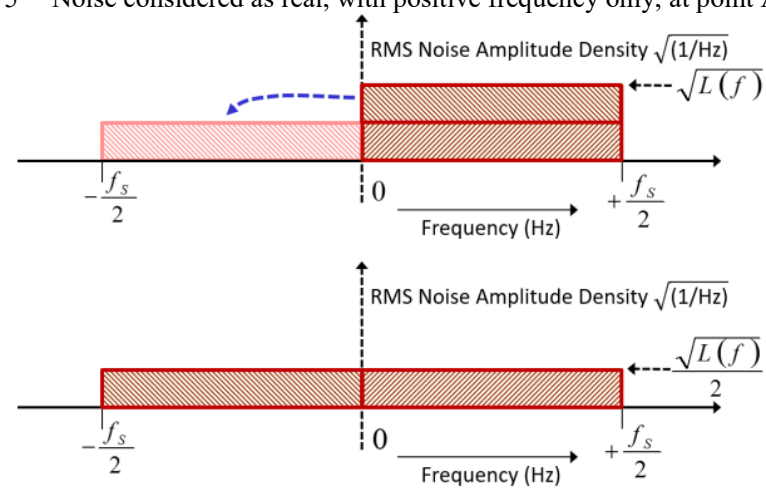

Fig. 6 Noise amplitude density split into complex components with positive and negative frequencies at point $B$

\section{B. De-correlation of noise through heterodyning}

However, the action of the heterodyning process (Fig. 7), with $f_{T}$ set to anything other than $0 \mathrm{~Hz}$, causes a de-correlation of the decomposed positive and negative frequency noise components. They are no longer at $\pm f_{N}$, but $-f_{N}-f_{T}$ and $+f_{N}-f_{T}$, so they cannot be considered to recombine by linear addition (e.g. by reversing (2) to (1)) to produce a single real sinusoid at frequency $f_{N}$, at the original amplitude at point $\mathrm{C}$ of Fig. 1. The decomposed and heterodyned components form a de-correlated set of exponentials across the range $-f_{S} / 2<f<+f_{S} / 2$, with relative amplitude density $\sqrt{L(f)} / 2$. The effect of aliasing also plays a part in the de-correlation. 


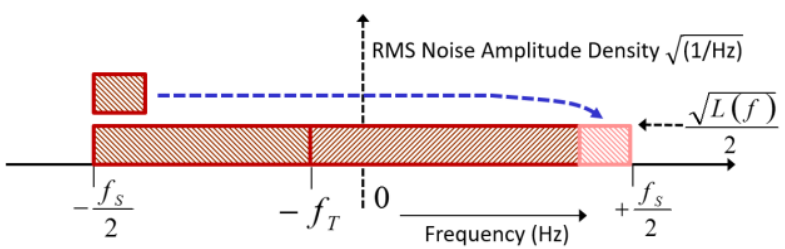

Fig. 7 De-correlation of complex noise components by heterodyning (and aliasing) at point $\mathrm{C}$

After the noise components have been de-correlated through heterodyning with $f_{T} \neq 0$, they must be recombined as the root-sum-of-squares (RSS) of the amplitudes.

\section{The noise level at point D of Fig. 1, just before filtering}

By considering the findings thus far, it is now possible to say that for a sinewave carrier with RMS amplitude 1 entering at point A of Fig. 1, the wanted component of it (heterodyned to $0 \mathrm{~Hz}$ ) which appears at point $\mathrm{D}$ will have an exponential (phasor) amplitude 1. At the same time, the exponential (phasor) amplitude density of noise, at all frequencies in the range $-f_{S} / 2<f<+f_{S} / 2$, will be $\sqrt{L(f)}$, because the system gain through the process from point A to point $D$, via (1) - (7), is identical for the carrier and all individual noise components, if $f_{T} \neq 0$.

\section{ERRORS DUE TO A SINGLE FREQUENCY COMPONENT AFTER HETERODYNING}

In this section, expressions are derived which can later form the kernels of integrations across frequency, in order to determine the overall RMS frequency error (FE) and ROCOF error (RFE) on the measurand $\mathbf{V}$ in Fig. 1.

Consider the real-valued input carrier at $+f_{C}$ with RMS amplitude 1 entering at point $\mathrm{A}$ of Fig. 1. After the heterodyning and filtering action of $H(f)$, the image component is rejected and the carrier emerges at points D and E of Fig. 1 as a complex exponential with magnitude 1 . Assuming $f_{T}=f_{C}$, the carrier at $\mathrm{E}$ has a constant phase that can be written as $\phi$.

Now consider any heterodyned component of noise at positive or negative frequency $f_{N}$ in Fig. 7 . The relative magnitude density of this complex exponential at point $\mathrm{D}$ of Fig. 1 will be will be $\sqrt{L(f)} \sqrt{1 / H z}$ as previously described. To analyse the effect of this on the measurements, we consider a small frequency segment of width $\delta f$ in the region of $f_{N}$, and consider the effect of the noise over $f_{N} \pm \delta f / 2$ to be concentrated at $f_{N}$. The magnitude of the resulting complex exponential, centred at $f_{N}$, can therefore be written as $M$, which essentially results in a modulation of the carrier.

$$
M=M(f)=\sqrt{L(f)} \delta f
$$

Since $L(f)$ is constant across $f$ for the special case of white noise, we can allocate a constant value $M$ which is valid for all $f$, and is not a function of $f$. However, between points $\mathrm{D}$ and $\mathrm{E}$ of Fig. 1 the modulation due to noise will be attenuated by $H(f)$. The above two paragraphs allow the following equation to be written which accounts for both the carrier and a single complex exponential due to a "spot" noise frequency in the region $-f_{S} / 2<f_{N}<f_{S} / 2$, over a small noise bandwidth $\delta f$ :

$$
\begin{aligned}
\mathbf{V} & =H\left(f_{C}-f_{T}\right) 1 e^{j\left(\phi+2 \pi\left(f_{C}-f_{T}\right) t\right)} \\
& +M H\left(f_{N}-f_{T}\right) e^{j 2 \pi\left(f_{N}-f_{T}\right) t}
\end{aligned}
$$

This simplifies by $(5)\left(\left(f_{C}-f_{T}\right)=0\right)$, and $H(0)=1$ :

$$
\mathbf{V}=1 e^{j \phi}+M H\left(f_{N}-f_{T}\right) e^{j 2 \pi\left(f_{N}-f_{C}\right) t}
$$

The examination of error is easiest done by considering the deviation of $\mathbf{V}$ from its "nominal" value of:

$$
\mathbf{V}_{\text {Nom }}=1 e^{j \phi}
$$

To do this, a new value $\Delta \mathbf{V}$ is defined by referencing $\mathbf{V}$ to $\mathbf{V}_{N o m}$, so that $\Delta \mathbf{V}$, in the presence of zero noise, would be a fixed phasor of value $(1+0 \mathrm{j}) . \Delta \mathbf{V}$ becomes a phasor of nominal value $(1+0 \mathrm{j})$, plus the interfering circular trajectory caused by the noise modulation:

$$
\begin{aligned}
\Delta \mathbf{V}= & \mathbf{V} / \mathbf{V}_{\text {Nom }} \\
\Delta \mathbf{V}= & 1+M H\left(f_{N}-f_{T}\right) e^{j\left(2 \pi\left(f_{N}-f_{C}\right) t-\phi\right)} \\
& \Delta \mathbf{V}=(1+0 \mathrm{j}) \text { plus a rotating phasor } \\
& \text { of amplitude } H\left(f_{N}-f_{T}\right) M \text { rotating at }\left(f_{N}-f_{C}\right) \mathrm{Hz}
\end{aligned}
$$

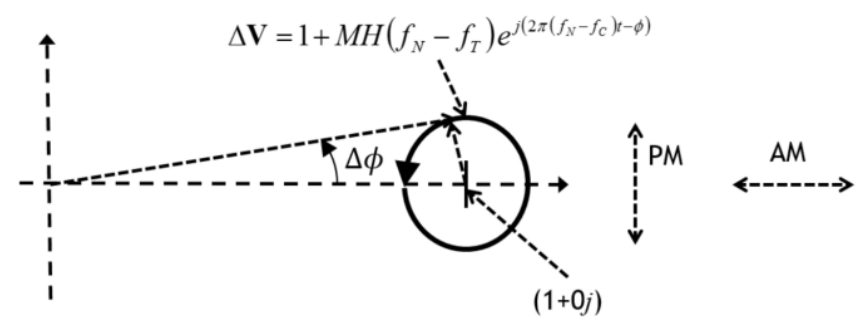

Fig. 8 Phasor $\Delta \mathbf{V}$ when a single noise or interfering component is added.

A phasor diagram representing $\Delta \mathbf{V}$ can be drawn as Fig. 8. $\Delta \mathbf{V}$ can be expanded:

$$
\begin{aligned}
\Delta \mathbf{V}= & 1+M H\left(f_{N}-f_{T}\right) \cos \left(2 \pi\left(f_{N}-f_{C}\right) t-\phi\right) \\
& +j M H\left(f_{N}-f_{T}\right) \sin \left(2 \pi\left(f_{N}-f_{C}\right) t-\phi\right) \\
\Delta \mathbf{V}= & +M H\left(f_{N}-f_{T}\right) \cos \left(2 \pi\left(f_{N}-f_{C}\right) t-\phi\right) \\
& +j M H\left(f_{N}-f_{T}\right) \cos \left(2 \pi\left(f_{N}-f_{C}\right) t-\pi / 2-\phi\right)
\end{aligned}
$$

This shows that every single complex exponential noise component, when considered individually, equates to both AM (Amplitude Modulation) and PM (Phase Modulation) of the carrier, simultaneously, with the PM effect lagging the AM by 90 degrees [5].

\section{A. Phase, Frequency and ROCOF error due to a single complex noise component}

To determine the frequency error resulting from an individual noise component, the assumption is made that the noise is small compared to the signal, so that $M<<1$, which is reasonable if SNR is in the usual ranges. In this case, the AM contributes nothing to the perception of phase on $\Delta \mathbf{V}$, but the PM component does $[5,6]$. 
The phase perturbation of $\boldsymbol{\Delta} \mathbf{V}$, due to just this single noise component, will be an amount $\Delta \phi$ which can be expressed as:

$$
\Delta \phi=M H\left(f_{N}-f_{T}\right) \sin \left(2 \pi\left(f_{N}-f_{C}\right) t-\phi\right)
$$

Evaluating the frequency error (FE) and ROCOF error (RFE) can now be done by differentiating phase and subsequently FE.

$$
\begin{aligned}
& \frac{d(\Delta \phi)}{d t}=2 \pi M\left(f_{N}-f_{C}\right) H\left(f_{N}-f_{T}\right) \cos \left(2 \pi\left(f_{N}-f_{C}\right) t-\phi\right) \\
& F E=\frac{1}{2 \pi} \frac{d(\Delta \phi)}{d t} \\
& F E=M\left(f_{N}-f_{C}\right) H\left(f_{N}-f_{T}\right) \cos \left(2 \pi\left(f_{N}-f_{C}\right) t-\phi\right) \\
& R F E=\frac{d}{d t}(F E) \\
& R F E=2 \pi M\left(f_{N}-f_{C}\right)^{2} H\left(f_{N}-f_{T}\right)\left(-\sin \left(2 \pi\left(f_{N}-f_{C}\right) t-\phi\right)\right)
\end{aligned}
$$

Therefore the amplitudes of FE and RFE, due to a single complex exponential noise or interfering component, are:

$$
\begin{aligned}
& F E_{R M S}=\frac{M}{\sqrt{2}}\left|\left(f_{N}-f_{C}\right) H\left(f_{N}-f_{T}\right)\right| \\
& R F E_{R M S}=\sqrt{2} \pi M\left|\left(f_{N}-f_{C}\right)^{2} H\left(f_{N}-f_{T}\right)\right|
\end{aligned}
$$

\section{OVERALL FE AND RFE FROM HETERODYNED MEASUREMENTS}

Following all the above arguments, it is now possible to write expressions for the total expected RMS FE and RFE after a heterodyned and filtered measurement of a single-phase real sinusoidal carrier.

The expression (31) for RMS FE is formed by examining the cumulative effect of every individual real noise signal component at frequency $f_{N}$, over the positive-frequency noise range $0<f_{N}<f_{S} / 2$, using (29) and (15) to determine the effect of each small segment of noise contribution $\delta f$, and being careful to use an RSS analysis since the noise components are de-correlated by the heterodyne process. The expression (32) for RMS RFE is similarly constructed using (30) and (15).

$$
\begin{aligned}
& F E_{R M S}=\frac{\sqrt{L(f)}}{\sqrt{2}} \sqrt{\int_{f_{N}=0}^{\frac{f_{S}}{2}}\left[\begin{array}{c}
\mid A\left(f_{N}-f_{C}\right) H\left(A\left(f_{N}-f_{T}\right)\right)^{2} \\
+\mid A\left(-f_{N}-f_{C}\right) H\left(A\left(-f_{N}-f_{T}\right)\right)^{2}
\end{array}\right] \cdot d f_{N}} \\
& R F E_{R M S}=\sqrt{2} \pi \sqrt{L(f)} \sqrt{\int_{f_{N}=0}^{\frac{f_{S}}{2}}\left[\begin{array}{c}
\left|\left(A\left(f_{N}-f_{C}\right)\right)^{2} H\left(A\left(f_{N}-f_{T}\right)\right)\right|^{2} \\
+\left(A\left(-f_{N}-f_{C}\right)\right)^{2} H\left(A\left(-f_{N}-f_{T}\right)\right)^{2}
\end{array}\right] \cdot d f_{N}}
\end{aligned}
$$

The function $A()$ in (31) and (32) refers to the potential aliasing of a sampled waveform onto a different frequency during the heterodyne process and can be evaluated as:

$$
A(f)=\left[\left(f+\frac{f_{S}}{2}\right) \bmod f_{S}\right]-\left(\frac{f_{S}}{2}\right)
$$

In (31) and (32), it is assumed that the heterodyned carrier image component at $\left(-f_{C}-f_{T}\right)$ is completely removed by the filtering. If this is not so, the "spectral leakage" term can result in large FE and RFE errors. On the assumption that the heterodyne stage is tuned so that $f_{T}=f_{C}$, then (31) and (32) can be shortened by writing:

$$
\begin{gathered}
\left\{\begin{array}{c}
f_{1}=A\left(f_{N}-f_{C}\right)=A\left(f_{N}-f_{T}\right) \\
\left.f_{2}=A\left(-f_{N}-f_{C}\right)=A\left(-f_{N}-f_{T}\right)\right\}
\end{array}\right. \\
\Rightarrow F E_{R M S}=\sqrt{\frac{L(f)}{2}} \sqrt{\int_{f_{N}=0}^{\frac{f_{S}}{2}}\left[\left|f_{1} H\left(f_{1}\right)\right|^{2}+\left|f_{2} H\left(f_{2}\right)\right|^{2}\right] \cdot d f_{N}} \\
\Rightarrow R F E_{R M S}=\pi \sqrt{2 L(f)} \sqrt{\int_{f_{N}=0}^{\frac{f_{S}}{2}}\left[\mid f_{1}^{2} H\left(\left.f_{1}\right|^{2}+\left|f_{2}^{2} H\left(f_{2}\right)\right|^{2}\right] \cdot d f_{N}\right.}
\end{gathered}
$$

The accurate expressions (35) and (36) have a slightly different form than those given in [5]. The most obvious difference is that the action of heterodyning is fully accounted for in (35) and (36), with an individual treatment of positive and negative frequencies, whereas [5] makes an approximation that $\left(f_{T}=f_{C}\right)<<f_{N}$. To better compare the predictions, modify (35) and (36) by adding the assumption that $\left(f_{T}=f_{C}\right)<<f_{N}$. In this case:

$$
\begin{aligned}
& F E_{R M S} \approx \sqrt{\frac{L(f)}{2}} \sqrt{\int_{f_{N}=0}^{\frac{f_{S}}{2}}\left[\left|f_{N} H\left(f_{N}\right)\right|^{2}+\left|-f_{N} H\left(-f_{N}\right)\right|^{2}\right] \cdot d f_{N}} \\
& R F E_{R M S} \approx \pi \sqrt{2 L(f)} \sqrt{\int_{f_{N}=0}^{\frac{f_{S}}{2}}\left[\left|f_{N}^{2} H\left(f_{N}\right)\right|^{2}+\left|\left(-f_{N}\right)^{2} H\left(-f_{N}\right)\right|^{2}\right] \cdot d f_{N}} \\
& \Rightarrow F E_{R M S} \approx \sqrt{L(f)} \sqrt{\int_{f_{N}=0}^{\frac{f_{S}}{2}}\left|f_{N} H\left(f_{N}\right)\right|^{2} \cdot d f_{N}} \\
& \Rightarrow R F E_{R M S} \approx 2 \pi \sqrt{L(f)} \sqrt{\frac{f_{S}}{2}}\left|f_{N}^{2} H\left(f_{N}\right)\right|^{2} \cdot d f_{N}
\end{aligned}
$$

Both (39) and (40) predict RMS FE and RFE errors which are half the magnitude of the expressions in [5].

\section{A. Three-phase heterodyned measurements}

The frequency and ROCOF of a set of $N$, independent, realvalued sinusoids with the same frequency can be determined by making $N$ independent measurements and averaging the results. If the sinusoids are independent, their noise is not correlated and the resulting FE and RFE will be reduced by $1 / \sqrt{ } N$. So, in the case of a three-phase measurement set, the expected errors will be (35) and (36) divided by $\sqrt{3}$ :

$$
F E_{3 \phi R M S} \approx \sqrt{\frac{L(f)}{3}} \sqrt{\int_{f_{N}=0}^{\frac{f_{S}}{2}}\left|f_{N} H\left(f_{N}\right)\right|^{2} \cdot d f_{N}}
$$




$$
R F E_{3 \phi R M S} \approx 2 \pi \sqrt{\frac{L(f)}{3}} \sqrt{\int_{f_{N}=0}^{\frac{f_{S}}{2}}\left|f_{N}^{2} H\left(f_{N}\right)\right|^{2} \cdot d f_{N}}
$$

In this paper, the expressions for errors contain integrations across the Nyquist range of frequencies, as continuous integral expressions. In practice, discrete numerical integration expressions are more convenient to evaluate. For example, the expression (42) can be approximated by choosing an interval $\Delta f<<f_{S}$ and then evaluating the following (or similar) using a computer, where $m_{2}=(m+1 / 2)$ to slightly enhance accuracy:

$R F E \approx 2 \pi \sqrt{\frac{L(f)}{3}} \sqrt{\left.\left(\sum_{m=0}^{\left[f_{s} / 2 \Delta f\right.}\right]^{-1}\right)\left|\left(m_{2} \Delta f\right)^{2} H\left(m_{2} \Delta f\right)\right|^{2} \cdot \Delta f}$

\section{Clarke (AND Park)TRansform Methods}

If a 3-phase set of signals is to be measured, which contains a dominant positive-sequence component, then the frequency and ROCOF can be measured directly using a Clarke transform approach. The dominant positive sequence signal set (with RMS magnitude A on each phase) is given by:

$$
\mathbf{V}_{\mathrm{abc}}=A_{R M S} \sqrt{2}\left[\cos (\phi) \cos \left(\phi-\frac{2 \pi}{3}\right) \cos \left(\phi+\frac{2 \pi}{3}\right)\right]^{T}
$$

The Clarke transform, which maps the three positivesequence signals onto the 2-dimensional vector $\mathbf{V}_{\boldsymbol{\alpha} \mathbf{\beta}}$, is:

$$
\mathbf{V}_{\alpha \beta}=\frac{2}{3 \sqrt{2}}\left[\begin{array}{ll}
1 & j
\end{array}\right]\left(\left[\begin{array}{ccc}
1 & -\frac{1}{2} & -\frac{1}{2} \\
0 & \frac{\sqrt{3}}{2} & -\frac{\sqrt{3}}{2}
\end{array}\right] \mathbf{V}_{\mathrm{abc}}\right)
$$

In (45), the Clarke transform gain is such that the signal set $\mathbf{V}_{\text {abc}}$, with the positive sequence having RMS magnitude $A_{R M S}$ on each phase, results in a steady-state value of $\left|\mathbf{V}_{\alpha \beta}\right|=A_{R M S}$. Meanwhile, the RMS noise on $\mathbf{V}_{\boldsymbol{\alpha} \beta}$ can be assessed directly by considering the RMS noise on $V_{a}, V_{b} \& V_{c}$ and how these noise components pass through (45).

There is no heterodyning (frequency translation) during the Clarke transformation process. Therefore, there is no decorrelation of either fundamental signal or noise components due to a frequency translation. Hence, an analysis of wideband noise contribution needs to consider only the positive-half of the frequency span $0<f_{N}<f_{S} / 2$, over which the relative RMS noise amplitude density is $\sqrt{L(f)} \sqrt{1 / H z}$ (Fig. 6 top). While the noise is correlated with itself between positive and negative frequencies on each of the three signals, the noise is not correlated between the three signals. So, the addition of the noise contributions from the three phases needs to be considered on an RMS basis.

The simplest way to describe the effect on frequency and ROCOF measurement is to consider the example snapshot in time when $\phi=0$. Other times could be used with the same result, but requiring a more complex mathematical description. At $\phi=0$, the expected result if the fundamentals have RMS amplitude 1, is $\mathbf{V}_{\alpha \beta}=1 \angle 0$. The expected measured phase of $\mathbf{V}_{\alpha \beta}$ is $\phi=0$. Any error in measuring this phase contributes to frequency and ROCOF error. The analysis proceeds with a similar argument as (23)-(30). Assuming the noise $L(f)<<1$, then the error on $V_{\alpha}=\operatorname{Re}\left(\mathbf{V}_{\alpha \beta}\right)$ contributes essentially nothing to the error. However, the RMS noise error in phase $\Delta \phi$ will be exactly equal to the RMS noise on $V_{\beta}=\operatorname{Im}\left(\mathbf{V}_{\alpha \beta}\right)$, i.e. $\Delta \phi=\angle \mathbf{V}_{\alpha \boldsymbol{\beta}} \approx V_{\beta}$, since $\angle \mathbf{V}_{\alpha \boldsymbol{\beta}} \approx V_{\beta}$ if $V_{\alpha} \approx 1$ and $V_{\beta}<<1$. On this basis, the noise $\Delta \phi$ can be expressed in a form, which also considers the filtering $H(f)$ :

$$
\Delta \phi=M \sin \left(2 \pi f_{N} t\right) \cdot H\left(f_{N}\right)
$$

The value for $M$ (valid across $0<f_{N}<f_{S} / 2$ ) is calculated via the RMS noise amplitude density on $V_{\beta}(45)$ :

$$
V_{\beta_{-} R M S}=\frac{2}{3 \sqrt{2}}\left(\sqrt{\left(\frac{\sqrt{3}}{2} \sqrt{L(f)}\right)^{2}+\left(\frac{\sqrt{3}}{2} \sqrt{L(f)}\right)^{2}}\right) \quad \sqrt{1 / H z}
$$

The value of $M$ is then determined by simplifying the peak error value of the error on $V_{\beta}$, and accounting for a finite frequency segment $\delta f$ :

$$
M=V_{\beta_{-} P k} \cdot \delta f=\sqrt{2} V_{\beta_{-} R M S} \cdot \delta f=\frac{\sqrt{2} L(f)}{\sqrt{3}} \cdot \delta f
$$

Finally, by a parallel and similar process to (16)-(30), and considering only $0<f_{N}<f_{S} / 2$ :

$$
\begin{aligned}
& F E_{\text {RMS Clarke }}=\sqrt{\frac{L(f)}{3}} \sqrt{\int_{f_{N}=0}^{\frac{f_{S}}{2}} \mid f_{N} H\left(f_{N}\right)^{2} \cdot d f_{N}} \\
& R F E_{\text {RMS Clarke }}=2 \pi \sqrt{\frac{L(f)}{3}} \sqrt{\int_{f_{N}=0}^{\frac{f_{S}}{2}} \mid f_{N}^{2} H\left(f_{N}\right)^{2} \cdot d f_{N}}
\end{aligned}
$$

In terms of performance against noise, (49) \& (50) show that the errors from a Clarke transform approach will be the same as the errors from averaged three-phase heterodyned measurements (41) \& (42), if the same filter is used. Additionally, should a Park transform be used instead of the Clarke transform, then so long as a quasi-static frequency estimate is used to define the rotating reference frame, the Park transform result sensitivities to noise will be identical to those of the Clarke transform. This is because the Park transform can be easily derived from a Clarke transform, by referring it to a rotating frame.

\section{OTHER METHODS}

\section{A. Use of Zero Crossings}

Compared to all but the simplest rectangular-windowed Heterodyne or Clarke/Park-transform methods, the results from zero-crossing measurements are noisy. This is because most of the waveform samples, including the samples with biggest amplitude and highest SNR, are not included in the analysis. 
Also, the options for filtering are limited. For example, taking a 1-cycle zero-crossing measurement, and averaging it with the next 1-cycle one, will produce an identical answer to that obtained by carrying out a 2-cycle analysis in the first place. This is because the zero-crossing time evaluated at the end of the $1^{\text {st }}$ cycle is shared and correlated between the two measurements. It is possible to update the measurement results every half cycle, using a rolling window of length $\mathrm{N}$ cycles, and allowing both positive-going and negative-going zerocrossings to bound the window.

A model to approximately predict FE and RFE from such zero-crossing methods has been developed. The noise correlation mechanisms are more complex than for heterodyned or Clarke/Park-transform measurements. The model is therefore lengthy to describe, even though it does not account for all the correlation mechanisms in perfect detail. Since the zero-crossing measurement technique is ultimately not as effective as the others, and due to space considerations, the model is not presented in this paper.

\section{B. Use of Phased-Locked-Loops (PLLs)}

PLLs have historically been used to synchronise sampling in (for example) power-quality analysers, by locking on to the mains frequency. PLLs are also commonly used within the control loops of power converters. However, their performance as frequency and ROCOF measurement devices is known to be relatively poor $[9,18]$. Most fundamentally, the closed loop of the PLL structure means that it responds as an infinite impulse response (IIR) device, with a damped resonance at some frequency. The IIR response deviates significantly from anything which could be related to a rectangular FIR window possessing low ENBW (Effective Noise Bandwidth) properties. The passband width is very wide compared to the effective latency. While not the focus of this paper, comparative results from 5 different PLL arrangements, shown in Table I, are presented. Equivalent performance can be realised by using a 3-phase PLL, or the average of 3 singlephase PLL outputs, if equivalent controller gains are applied. Therefore, while 5 PLL configurations were considered, there are only three distinct results, named P1, P2 \& P3 in Table I. We used single-phase PLLs and three-phase PLLs from the MATLAB $®$ "Simscape Power Systems" blockset (present and past versions). P1 and P2 have controllers of the "PID" variety with a differential damping term. P3 is the classic "Type 2" PLL containing a "PI" controller. "Type 3" PLLs which include controller terms in $\mathrm{s}^{-2}$ were not explicitly examined since their response to dynamic events such as phase steps (a common occurrence in power systems) is reported to be even worse than "Type 2" [19]. All these PLLs also contain a singlecycle tunable boxcar filter within the control loop, and an additional 2-pole low-pass filter with $f_{\mathrm{C}}=25 \mathrm{~Hz}$ and $\zeta=0.707$, which post-filters the PLL loop frequency before output. Ramp-rate filters were disabled since these nonlinear devices can impart bias on the output in the presence of inter-harmonic components.
TABLE I PLL EXAMPLES USED FOR COMPARISON

\begin{tabular}{|l|c|c|c|}
\hline & $\begin{array}{c}\text { Controller } \\
\text { Kp, Ki, Kd }\} \text { and } \tau \\
C(s)=K_{P}+\frac{K_{I}}{s}+\frac{K_{D} s}{(1+\tau s)}\end{array}$ & $\begin{array}{c}\text { Resonant } \\
\text { peak }\end{array}$ & $\begin{array}{c}\text { Gain \& } \\
\text { Phase } \\
\text { Margins }\end{array}$ \\
\hline P1 : 3-Phase PLL & $\{180,3200,1\}$ and 0.0001 & $4 \mathrm{~dB} 27 \mathrm{~Hz}$ & $10 \mathrm{~dB}, 40^{\circ}$ \\
\hline P2 : 3-Phase PLL & $\{90,1600,0.5\}$ and 0.0001 & $2 \mathrm{~dB} 6 \mathrm{~Hz}$ & $16 \mathrm{~dB}, 56^{\circ}$ \\
\hline P2 : 3x 1-Phase PLLs & $\{180,3200,1\}$ and 0.0001 & $2 \mathrm{~dB} 6 \mathrm{~Hz}$ & $16 \mathrm{~dB}, 56^{\circ}$ \\
\hline P3 : 3-Phase PLL & $\{60,1400,0\}$ & $11 \mathrm{~dB} 18 \mathrm{~Hz}$ & $4 \mathrm{~dB}, 18^{\circ}$ \\
\hline P3 : 3x 1-Phase PLLs & $\{120,2800,0\}$ & $11 \mathrm{~dB} 18 \mathrm{~Hz}$ & $4 \mathrm{~dB}, 18^{\circ}$ \\
\hline
\end{tabular}

\section{LATENCY OF THE METHODS}

The latency of the heterodyned and Clarke/Park-transform methods (excluding calculations and communications) is equal to half the filter window time length. Since the measurements are not tied to zero crossings, a new measurement output can be produced every time a new sample arrives, and the practical measurement latency can be close to the theoretical value.

By comparison, a zero-crossing frequency measurement obtained across a base window of $N$ cycles is constrained, in that it can only produce a new result when a zero crossing occurs. This means that the latency varies with time in a sawtooth fashion. Additionally, to derive ROCOF from the frequency requires differentiation using 2 samples obtained from the base window at the update rate, which could be every 1 or $1 / 2$ cycle. Table II describes the options for latency.

TABLE II COMPARISON OF LATENCY OF FREQUENCY AND ROCOF MEASUREMENTS, FOR A BASE WINDOW OF TIME LENGTH N CYCLES

\begin{tabular}{|c|c|c|c|}
\hline & $\begin{array}{c}\text { Minimum } \\
\text { latency }\end{array}$ & $\begin{array}{l}\text { Maximum } \\
\text { Latency }\end{array}$ & $\begin{array}{l}\text { Average } \\
\text { Latency }\end{array}$ \\
\hline $\begin{array}{c}\text { Heterodyne: } \\
\text { Frequency and ROCOF }\end{array}$ & $N / 2$ & $N / 2$ & $N / 2$ \\
\hline $\begin{array}{l}\text { Clarke/Park transform: } \\
\text { Frequency and ROCOF }\end{array}$ & $N / 2$ & $N / 2$ & $N / 2$ \\
\hline $\begin{array}{c}\text { Zero crossing, update every cycle: } \\
\text { Frequency }\end{array}$ & $N / 2$ & $N / 2+1$ & $N / 2+1 / 2$ \\
\hline $\begin{array}{l}\text { Zero crossing, update every cycle: } \\
\text { ROCOF }\end{array}$ & $(N+1) / 2$ & $(N+1) / 2+1$ & $N / 2+1$ \\
\hline $\begin{array}{c}\text { Zero crossings, update every } 1 / 2 \\
\text { cycle: } \\
\text { Frequency }\end{array}$ & $N / 2$ & $N / 2+1 / 2$ & $N / 2+1 / 4$ \\
\hline $\begin{array}{c}\text { Zero crossings, update every } 1 / 2 \\
\text { cycle: } \\
\text { ROCOF }\end{array}$ & $(N+1 / 2) / 2$ & $(N+1 / 2) / 2+1 / 2$ & $N / 2+1 / 2$ \\
\hline
\end{tabular}

The useful latency of the frequency and ROCOF measurements at a PLL output are significant, but hard to quantify, due to the IIR oscillatory nature of the impulse and step responses in the time domain. While the group delay evaluated from the frequency-domain response is relatively low at $\sim 0.02 \mathrm{~s}$, the real-world latency for designs P1-P3 in Table I is estimated from the time-domain response in this paper as 5 cycles $(100 \mathrm{~ms})$. 


\section{SELECTING THE BEST FILTER WINDOW FOR FREQUENCY AND ROCOF MEASUREMENTS}

While (35)-(42) and (49)-(50) provide useful mathematical expressions through which to predict the error magnitudes, it is also illuminating to perform a more intuitive analysis, which leads to a fundamental "prediction" of the best filter/windows to use for frequency ROCOF measurements. It is well known that in the presence of white noise spread evenly across the whole Nyquist band (e.g. Fig. 6 lower), the lowest-noise direct measurement of signal amplitude or phase, for a specified window length, would be achieved by using a rectangular boxcar window, since it has the lowest ENBW [20]. However, the frequency measurement requires differentiation of phase. In practice, the differentiation can be implemented digitally over a short 2-sample window, but, for the sake of understanding only (not the actual measurement algorithm), can here be approximated by the continuous-time Laplace operator $s$.

The differentiation is a part of the measurement filter chain. If this chain is linear, then the order of the filter components can be adjusted without affecting the final result. So, instead of being the last part of the chain, the differentiation stage can be considered to apply before the main filter windowing, at point $\mathrm{C}$ or D in Fig. 1. On that basis, the differentiation step applies a colouring to the noise, through the application of $s=j 2 \pi f$. Essentially the noise can be considered to be modified to a shape such as Fig. 9.

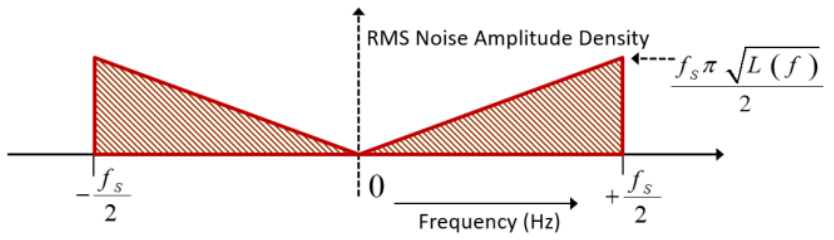

Fig. 9 Noise coloured by a single differentiation $s=j 2 \pi f$.

Logic would then dictate that the lowest noise output would then be obtained by de-colouring the noise by a filter with a response equal to $(1 / s)$ (rolloff $20 \mathrm{~dB} /$ decade), and then following with a rectangular boxcar window which has the lowest ENBW for white noise. The de-colouring filter $(1 / s)$ cannot be exactly implemented, since it is an unbounded integration. However, a bounded integration over finite time can be implemented. It is, of course, a rectangular boxcar window with a rolloff of $20 \mathrm{~dB} /$ decade. This suggests that while a single boxcar filter has the best ENBW for a normal measurement, when the measurement result is differentiated, the best filter will be a cascade of two boxcar filters, with a rolloff of $40 \mathrm{~dB} /$ decade, and intuition would suggest that the filtering is likely to be the most effective if the boxcars are of equal length. A similar filter with $\sim 40 \mathrm{~dB} /$ decade rolloff should also give good performance.

The same argument can be extended to the ROCOF measurement, which requires 2 stages of differentiation, by $s^{2}$. This colours the noise even more (Fig. 10), as the noise rises at $40 \mathrm{~dB} /$ decade. However the colouring can be largely "undone" by applying 2 boxcars, which have a combined rolloff of
$40 \mathrm{~dB} /$ decade. Added to the single boxcar required to provide the main filter, this suggests that the best filter to use for double-differentiated measurements like ROCOF is likely to be a cascade of 3 equal-length boxcar filters, with a total rolloff of $60 \mathrm{~dB} /$ decade. Other filters with the same $\sim 60 \mathrm{~dB} /$ decade rolloff will also be competitive.

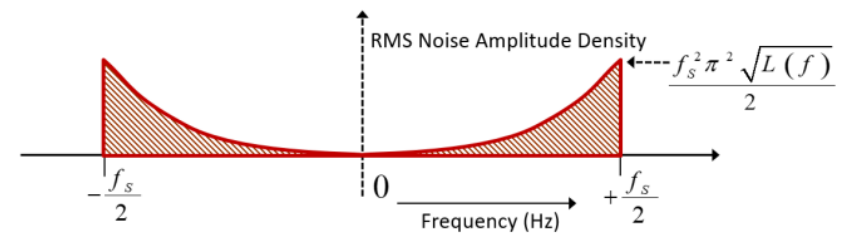

Fig. 10 Noise coloured by a double differentiation $s^{2}=-4 \pi^{2} f^{2}$.

\section{GAUSSIAN VS QUANTISATION NOISE}

The predictions of errors for Heterodyned and Clarke/Parktransform methods, using the methods in sections $\mathrm{V}$ and 0 , assumes that each sample is subject to Gaussian (white) noise that is uncorrelated between samples. The validity of this assumption depends upon whether the noise is composed of genuine white noise (e.g. from analogue circuitry, imperfect ADC linearity, or deliberate ADC dithering), or ideal quantisation errors due to perfectly linear ADC behaviour. In reality, front-end noise will be composed of a mixture of the two. For Heterodyned and Clarke/Park-transform methods, the actual FE and RFE is found to be mostly independent of the exact format of the noise, since there are many samples per cycle. However, a special case can occur if the noise is dominated by ADC quantisation (including static INL and DNL performances), with negligible analogue noise contribution, and the sample rate is an exact multiple of the signal fundamental frequency, and the input signal waveform is entirely steady-state. In this corner case the noise can concentrate at particular frequencies [16, 17]. If the digital filter places a zero near any of those frequencies, the noise can be highly attenuated, and errors reduced. However, in practice this scenario is highly unlikely to occur, and, if it does, the errors are reduced, not increased.

The errors from zero-crossing methods are much more dependent on the exact scenario, due to the small number of samples used and potential correlations. The predictions and simulations for FE and RFE from zero-crossings, for the same SNR and sample rate, show a strong dependence on exactly how the noise is split between white and quantisation types, the fundamental frequency, and the precise time of the measurement.

\section{COMPARISON OF PREDICTIONS AGAINST SIMULATIONS}

To compare the predictions against reality, fundamental signals at frequencies close to $50 \mathrm{~Hz}$ were synthesised at a $10 \mathrm{kHz}$ sample rate. Noise was added to the signals representing $\mathrm{SNR}=88.2 \mathrm{~dB}$. The simulations were carried out twice for each algorithm/window/filter and frequency: firstly using white noise, and then again using noise which is due to perfect linear quantisation, and contains more (but probably inconsequential in a practical application) quantisation 
correlations. All the algorithms are coded in Simulink/C so that they can be built/compiled for execution in real-time on suitable target platforms. However, the results shown are derived from desktop simulations, and presented in Table III.

The agreement between predictions and simulations is reasonable, typically within $10 \%$ and often less than $5 \%$. The performance of the three-phase Clark-transform algorithm is shown to be exactly equivalent to the three-phase heterodyned measurements, for equivalent filtering, as predicted. In general, there is little marked difference between results using white noise, and results using purely quantisation noise. However, there are two exceptions from these generalisations:

1) The FE, and particularly RFE, for heterodyned and Clarke/Park transform methods, is lower than predicted by up to $35 \%$ when the shortest single-cycle single-boxcar window is used. This is probably because each digital differentiation uses a 2 -sample window and provides a tiny bit of additional filtering. These same single-boxcar measurements possess large FE and RFE, and are not recommended for frequency or ROCOF assessment.

2) Zero errors can be recorded for algorithms which use a single boxcar filter of length 2 cycles, or the 2-cycle zerocrossing algorithm, for $\mathrm{f}_{\mathrm{C}}=50.1253 \mathrm{~Hz}$, when the noise is modelled as perfectly quantised. This is due to perfect correlation of the quantisation noise, as discussed above, and is unlikely to be observed in practice.

To further examine the effects of algorithm and filtering options, predictions and simulations are carried out for the three-phase algorithms using a wider range of window lengths (up to 12 cycles) and filter designs, at the single frequency $f_{C}=50.033 \mathrm{~Hz}$. The predicted errors continue to match the simulations, generally within $5 \%$, with occasional outliers. All the simulated results are summarised on Fig. 11 (FE) and Fig. 12 (RFE). The Clarke/Park and heterodyned measurements are labelled with the cascaded boxcar lengths, in cycles, within \{\} brackets. The zero-crossing measurements are labelled $\mathrm{Zn}: 1$ and $\mathrm{Zn}: 1 / 2$, where $\mathrm{n}$ is the base window length in cycles, and the 1 or $1 / 2$ signifies a 1 -cycle or $1 / 2$-cycle update rate.

Fig. 11 verifies that for a given latency, the best results are always obtained by using a Clarke/Park or heterodyned measurement using a filter which consists of 2 cascaded boxcars, of roughly equal lengths, with a rolloff of $\sim 40 \mathrm{~dB} /$ decade. For the longest 12-cycle window, some 3-stage filters with very unequal boxcar lengths such as $\{6.5,5.9,0.1\}$, $\left\{6,5 \frac{1}{2}, 1 / 2\right\}$ etc. provide almost equivalent performance to the 2 stage $\{6,6\}$ filter. This shows that there can be some careful deviation from the "optimal" design without incurring serious penalties. However, moving to a 3 (or more) stage filter using roughly equal-length boxcars, with $>=60 \mathrm{~dB} /$ decade rolloff, leads to noticeably worse results. The PLLs evaluated, (P1, P2 \& P3 from Table I) all perform poorly. Even if their latency was considered to be half of the estimated value ( 5 cycles), their performance would still not be competitive.

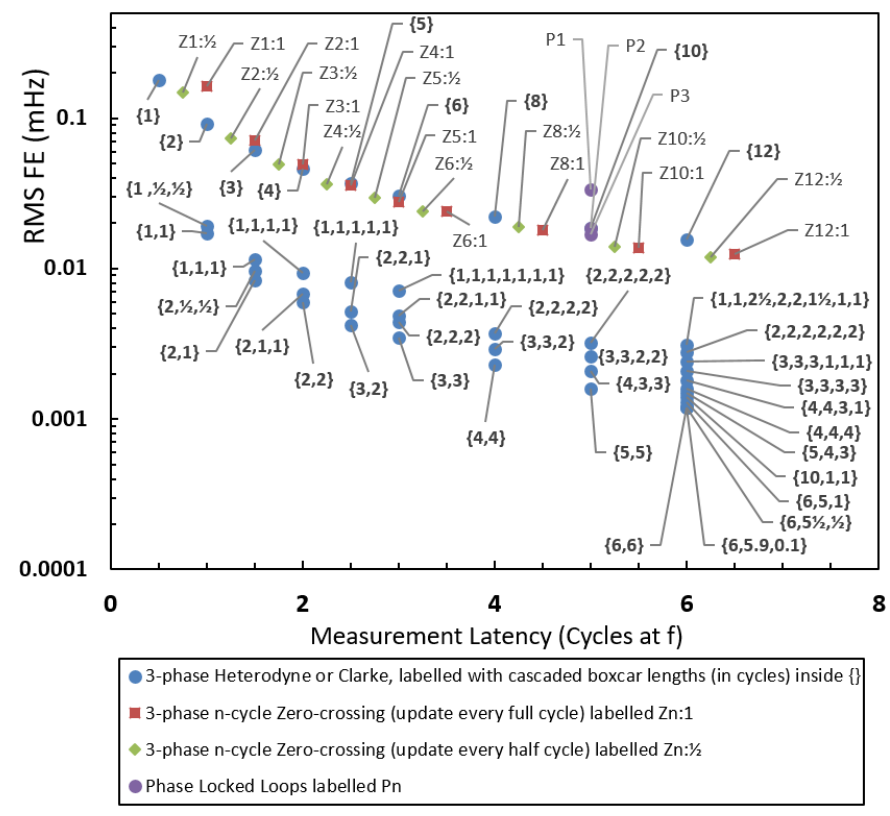

Fig. 11 RMS Frequency errors obtained by simulation, for three-phase measurements, using different algorithms and window lengths between 1 and 12 cycles. $f_{C}=50.033 \mathrm{~Hz}$. $f_{S}=10 \mathrm{kHz}$. SNR $=88.2 \mathrm{~dB} L(f)=-125.17 \mathrm{dBc} / \mathrm{Hz}$. All noise modelled as white (Gaussian).

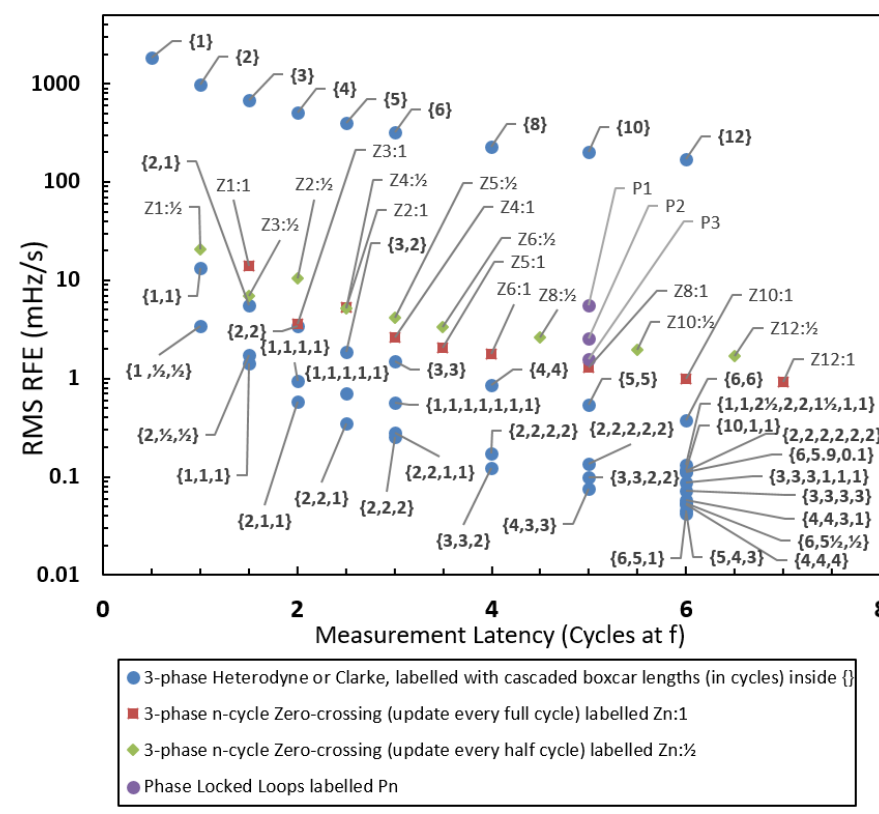

Fig. 12 RMS RFE errors obtained by simulation, for three-phase measurements, using different algorithms and window lengths between 1 and 12 cycles. $f_{C}=50.033$ Hz. $f_{S}=10 \mathrm{kHz}$. SNR=88.2 dB $L(f)=-125.17 \mathrm{dBc} / \mathrm{Hz}$. All noise modelled as white (Gaussian).

Similarly, Fig. 12 shows that the best results in terms of ROCOF are always obtained by Clarke/Park or heterodyned measurements which use a filter made up of a cascade of 3 boxcar filters of roughly equal lengths, or a filter which has similar performance, possessing rolloff of $\sim 60 \mathrm{~dB} /$ decade. Clarke/Park or heterodyne measurements using fewer cascaded boxcar filters produce significantly worse results, as do zerocrossing measurements. Moving to a 4 (or more) stage filter 
using roughly equal-length boxcars, with $\geq 80 \mathrm{~dB} /$ decade rolloff, also leads to noticeably worse results.

\section{CONCLUSIONS AND DISCUSSION}

The formulas derived in this paper allow prediction of the RMS errors on frequency and ROCOF measurements, due to white noise, for a particular sample rate, SNR, and algorithm/window/filter configuration. SNR needs to be carefully assessed, accounting for pre-ADC analogue instrumentation noise, $\mathrm{ADC}$ quantisation, $\mathrm{ADC}$ aperture jitter, ADC dithering (if applied), and ADC scaling. Knowledge of the mechanisms by which noise percolates to the final measurements also allows a rational prediction of the "optimal" filters for such measurements, in terms of performance against white noise. Predictions and simulations show that for frequency measurement, the best measurements are made with heterodyned or Clarke/Park-transform based measurements which use a filter that consists of a cascade of 2 roughly-equallength boxcar filters, or a similar filter possessing roughly $40 \mathrm{~dB} /$ decade rolloff. The best filters for ROCOF, however, require $\sim 60 \mathrm{~dB} /$ decade filtering, which can be achieved using a cascade of 3 roughly-equal-length boxcar filters.

It is possible to extrapolate from the presented results using (41)-(42) and (49)-(50) as guidelines. In an application with SNR higher or lower than that presented, every $6 \mathrm{~dB}$ increase of SNR halves the FE and RFE errors, and vice versa. The effect of sample rate needs careful consideration. In a conventional un-differentiated measurement application, increasing sample rate always decreases the effect of noise on the final measurement, since the linear noise amplitude density $\sqrt{ } L(f)$ scales with $1 / \sqrt{ } f_{S}$, as the noise is spread over a wider Nyquist band. Any sensible filter $H(f)$ with rolloff bigger than $0 \mathrm{~dB} /$ decade normally allows a higher-sample rate application to reject a higher proportion of the noise. However, the differentiated FE and RFE errors due to noise are additionally affected by the presence of $f_{N}$ and $f_{N}^{2}$ in the integral kernels of (for example) (49) \& (50). To realise a measurement whose error due to noise reduces with increasing sample rate, it is necessary for the rolloff in $H(f)$ to be more than $20 \mathrm{~dB} /$ decade for a frequency measurement, and more than $40 \mathrm{~dB} /$ decade for a ROCOF measurement. Since the recommended filters (for noise) have rolloffs of $40 \mathrm{~dB} /$ decade for frequency and $60 \mathrm{~dB} /$ decade for ROCOF, these conditions should be met in a well-designed application. So, as usual, sample rate should be kept as high as reasonably possible to minimise the effect of noise. Down-sampling to lower rates should be implemented as late in the signal processing chain as possible. However, if this is not possible, even simple front-end over-sampling can be beneficial [21].

Some applications down-sample the phase measurand to a lower sample rate (e.g. $50 \mathrm{~Hz}$ ), and then deduce frequency and ROCOF using 2-sample finite difference equations at the lower sample rate. In this case, the output of each low-rate finite difference equation is equivalent to differentiation at the original (higher) sample rate, combined with a boxcar filter at the higher sample rate, with the boxcar length equal to the lower-sample-rate period. For these applications, this is an important consideration when designing the core (higher sample rate) filter. Many existing algorithms apply extra filtering in this manner, perhaps without realising it, but sometimes to good practical effect.

Zero-crossing and PLL-based methods cannot compete against heterodyned and Clark-transform methods, if sensible filters are selected for them.

In a real application, overall filter design will be defined not only by performance requirements against white noise, but also by other requirements such as passband flatness, and general stop-band rejection of specific signals like harmonics and lowfrequency inter-harmonics. For example the requirements for PMUs [22, 23] contains strict requirements for FE due to outof-band signal application. In $[12,24]$ this led to a filter design with 6 cascaded boxcar filters in the frequency measurement path, and 7 in the ROCOF path, possessing roll-offs of $\sim 120$ and $\sim 140 \mathrm{~dB} /$ decade. We now know that such filters are far from ideal in terms of white noise performance. A design with fewer (but longer) stages might perform better, if it could also meet the other system requirements. Discussions in [6] described how the optimal filters to measure phasors, frequency and ROCOF may well be quite different to each other. Work in this paper reinforces that message.

\section{REFERENCES}

[1] Reactive Technologies, "GridMetrix," Available: https://www.reactivetechnologies.com, accessed Sept 2017.

[2] R. Ghiga, K. Martin, Q. Wu, and A. Nielsen, "Phasor Measurement Unit Test under Interference Conditions," IEEE Transactions on Power Delivery, 2017.

[3] D. Macii, D. Fontanelli, D. Petri, and G. Barchi, "Impact of Wideband Noise on Synchrophasor, Frequency and ROCOF Estimation," in IEEE AMPS (Applied Measurements for Power Systems), Aachen, 2015, p. 6.

[4] D. Macii, D. Fontanelli, G. Barchi, and D. Petri, "Impact of Acquisition Wideband Noise on Synchrophasor Measurements: A Design Perspective," IEEE Transactions on Instrumentation and Measurement, vol. 65 , pp. 2244-2253, Oct 2016.

[5] B. Dickerson, "Effect of PMU Analog Input Section Performance on Frequency and ROCOF Estimation Error," in IEEE AMPS (Applied Measurements for Power Systems), Aachen, 2015, p. 6.

[6] A. J. Roscoe, B. Dickerson, and K. E. Martin, "Filter design masks for C37.118.1a-compliant frequency-tracking and fixed-filter M-class Phasor Measurement Units (PMUs)," IEEE Transactions on Instrumentation and Measurement, vol. 64, pp. 2096-2107, 2015.

[7] E. Jacobsen and R. Lyons, "The sliding DFT," IEEE Signal Processing Magazine, vol. 20, pp. 74-80, Mar 2003.

[8] A. J. Roscoe and S. M. Blair, "Choice and Properties of Adaptive and Tunable Digital Boxcar (Moving Average) Filters for Power Systems and other Signal Processing Applications," in IEEE Applied Measurements in Power Systems (AMPS), Aachen, Germany, 2016.

[9] A. J. Roscoe, G. M. Burt, and J. R. McDonald, "Frequency and fundamental signal measurement algorithms for distributed control and protection applications," IET Generation, Transmission \& Distribution vol. 3, pp. 485-495, May 2009

[10] A. J. Roscoe, R. Carter, A. Cruden, and G. M. Burt, "Fast-Responding Measurements of Power System Harmonics using Discrete and Fast Fourier Transforms with Low Spectral Leakage," in 1st IET Renewable Power Generation Conference, Edinburgh, Scotland, 2011.

[11] A. J. Roscoe, S. M. Blair, and G. M. Burt, "Benchmarking and optimisation of Simulink code using Real-Time Workshop and Embedded Coder for inverter and microgrid control applications," in 
UPEC: 2009 44th International Universities Power Engineering Conference, 2009, pp. 532-536.

[12] A. J. Roscoe, I. F. Abdulhadi, and G. M. Burt, "P and M Class Phasor Measurement Unit Algorithms using Adaptive Cascaded Filters," IEEE Transactions on Power Delivery, vol. 28, pp. 1447-1459, 2013.

[13] B. Bannon, "Sampled Systems and the Effects of Clock Phase Noise and Jitter," Analogue Devices, AN-756, 2004. Available:

http://www.analog.com/media/en/technical-documentation/applicationnotes/AN-756.pdf, accessed Sept 2017.

[14] Analog Devices Inc., "AD7863: Simultaneous Sampling Dual 175 kSPS 14-Bit ADC," 2006 Available: http://www.analog.com/media/en/technical-documentation/datasheets/AD7863.pdf, accessed Nov 2017.

[15] X. D. Zhao, D. M. Laverty, A. McKernan, D. J. Morrow, K. McLaughlin, et al., "GPS-Disciplined Analog-to-Digital Converter for Phasor Measurement Applications," IEEE Transactions on Instrumentation and Measurement, vol. 66, pp. 2349-2357, Sep 2017.

[16] "Chapter 2," in Analog-Digital Conversion, ISBN 0-916550-27-3, W. Kester, Ed.: Analog Devices Inc., 2004. Available: http://www.analog.com/en/education/education-library/data-conversionhandbook.html.

[17] "Chapter 2," in The Data Conversion Handbook, ISBN 0-7506-7841-0, W. Kester, Ed.: Newnes, 2005.

[18] A. Giles, A. J. Roscoe, and O. Anaya-Lara, "Appropriate Means of Frequency Measurement for the Provision of Inertial Response From a Wind Turbine," in ASRANet International Conference on Offshore Renewable Energy, Glasgow, UK, 2014. Available: http://strathprints.strath.ac.uk/49545/, accessed.

[19] S. Golestan, M. Monfared, F. D. Freijedo, and J. M. Guerrero, "Advantages and Challenges of a Type-3 PLL," IEEE Transactions on Power Electronics, vol. 28, pp. 4985-4997, Nov 2013.

[20] F. J. Harris, "Use of Windows for Harmonic-Analysis with Discrete Fourier-Transform," Proceedings of the IEEE, vol. 66, pp. 51-83, 1978.

[21] L. W. Zhan, Y. Liu, J. Culliss, J. Y. Zhao, and Y. L. Liu, "Dynamic Single-Phase Synchronized Phase and Frequency Estimation at the Distribution Level," IEEE Transactions on Smart Grid, vol. 6, pp. 20132022, Jul 2015.

[22] IEEE, "IEEE Standard for Synchrophasor Measurements for Power Systems," C37.118.1-2011, 2011.

[23] IEEE, "IEEE Standard for Synchrophasor Measurements for Power Systems -- Amendment 1: Modification of Selected Performance Requirements " C37.118.1a-2014, 2014.

[24] A. J. Roscoe, "Exploring the relative performance of frequency-tracking and fixed-filter Phasor Measurement Unit algorithms under C37.118 test procedures, the effects of interharmonics, and initial attempts at merging P class response with M class filtering," IEEE Transactions on Instrumentation and Measurement, vol. 62, pp. 2140-2153, 2013. 


\section{BIOGRAPHIES}

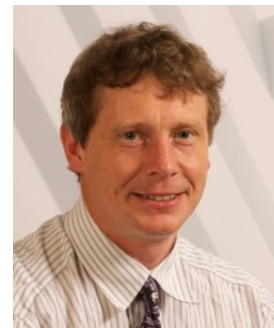

Andrew J. Roscoe (M'13-SM'15) received the B.A. and M.A. degree in Electrical and Information Sciences Tripos at Pembroke College, Cambridge, England in 1991 \& 1994. Andrew worked for GEC Marconi from 1991 to 1995 , where he was involved in antenna design and calibration, specialising in millimetre wave systems and solid-state phasedarray radars. Andrew worked from 1995 to 2003 with Hewlett Packard and subsequently Agilent Technologies, in the field of microwave communication systems, specialising in the design of test and measurement systems for personal mobile and satellite communications. Since 2004, Andrew has been working at the University of Strathclyde in the field of power systems. His specialist areas include power system measurement algorithms, marine power systems, laboratory demonstration with power-hardware-in-the loop capability, and the integration of high penetrations of converter-connected renewables.

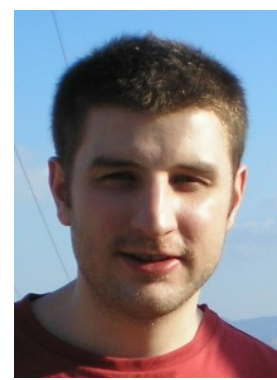

Steven M. Blair (M'09) received the M.Eng. degree (with distinction) in computer and electronic systems and the Ph.D. degree in electrical engineering from the University of Strathclyde, Glasgow, UK in 2008 and 2013, respectively.

Dr Blair is presently a Research Fellow at the Institute for Energy and Environment, Department of Electronic and Electrical Engineering, University of Strathclyde. He is a member of IEC Technical Committee 57 Working Group 10 and CIGRE Working Group B5.64. His research interests include power system protection, communications, measurements, power quality, real-time simulation, and fault current limitation.

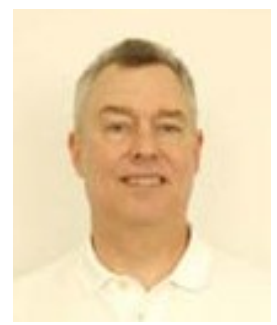

Bill Dickerson (M'85-F'17) is Chairman of the Board of Arbiter Systems, Inc. in Paso Robles, California. Bill received his BSEE from Washington University in St. Louis in 1975 and his Masters in Business Administration from the University of Michigan in 1979. Bill worked at Hewlett-Packard Company in Palo Alto, California and Spokane, Washington from 1979 until 1986, when he and his current partners bought Arbiter Systems, Inc.

Arbiter Systems is the industry leader in precise measurements for power systems and in accurate, reliable timing products for the power industry. Bill was the original product designer for Arbiter's GPS timing product line and the Model 1133A Power Sentinel, the industry's most accurate Phasor Measurement Unit (PMU).

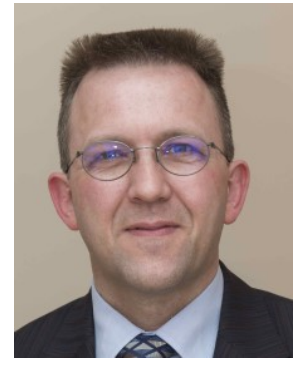

Gert Rietveld (M'10-SM'12) was born in Hardinxveld-Giessendam, The Netherlands, in 1965. He received the M.Sc. (cum laude) and $\mathrm{Ph} . \mathrm{D}$. degrees in low-temperature and solid-state physics from the Delft University of Technology, Delft, The Netherlands, in 1988 and 1993 respectively.

In 1993, he joined VSL, Delft, where he is a Chief Metrologist with the Research an Development Department. His current research interests cover the breadth of electromagnetic precision measurements, focusing on power and high-voltage measurement systems and electrical quantum standards.

Dr. Rietveld is a member of the International Committee for Weights and Measures and the President of its Consultative Committee for Electricity and Magnetism (CCEM). He is also the Chair of the Energy Task Group of the European Association of the National Metrology Institutes (EURAMET), Founding Chair of the EURAMET Subcommittee on Power and Energy, and a member of several CCEM, EURAMET, CIGRE, and IEEE Working Groups. 


\section{APPENDIX 1 : COMPARISONS OF PREDICTIONS AGAINST SIMULATIONS}

TABLE III RMS FE AND RFE ERRORS FROM NUMERIC SIMULATIONS, AGAINST PREDICTIONS (IN BRACKETS). $F_{S}=10 \mathrm{KHZ} . \mathrm{SNR}=88.2 \mathrm{DB} L(F)=-125.17 \mathrm{DBC} / \mathrm{HZ}$. 'Q' : NOISE MODELLED AS PURELY QUANTISATION. 'W' : NOISE MODELLED AS WHITE (GAUSSIAN). '*' SIGNIFIES AVERAGE LATENCY.

\begin{tabular}{|c|c|c|c|c|c|c|c|c|c|c|}
\hline & \multicolumn{4}{|c|}{ Filter } & \multicolumn{2}{|c|}{$f_{C}=50.033$} & \multicolumn{2}{|c|}{$f_{C}=50.0626$} & \multicolumn{2}{|c|}{$f_{C}=50.1253$} \\
\hline Method & $\begin{array}{c}\text { Number } \\
\text { of } \\
\text { boxcar } \\
\text { sections }\end{array}$ & $\begin{array}{l}\text { Length } \\
\text { of each } \\
\text { boxcar } \\
\text { (cycles) }\end{array}$ & $\begin{array}{c}\text { Total } \\
\text { window } \\
\text { length } \\
\text { (cycles) }\end{array}$ & $\begin{array}{c}\text { Latency } \\
\text { (cyles) }\end{array}$ & $\begin{array}{c}\text { RMS } \\
\text { FE } \\
(\mathrm{mHz})\end{array}$ & $\begin{array}{c}\text { RMS } \\
\text { RFE } \\
(\mathrm{mHz} / \mathrm{s})\end{array}$ & $\begin{array}{c}\text { RMS } \\
\text { FE } \\
(\mathrm{mHz})\end{array}$ & $\begin{array}{c}\text { RMS } \\
\text { RFE } \\
(\mathrm{mHz} / \mathrm{s})\end{array}$ & $\begin{array}{c}\text { RMS } \\
\text { FE } \\
(\mathrm{mHz})\end{array}$ & $\begin{array}{c}\text { RMS } \\
\text { RFE } \\
(\mathrm{mHz} / \mathrm{s})\end{array}$ \\
\hline 1 phase Heterodyne & 1 & $\{1\}$ & 1 & $1 / 2$ & $\begin{array}{c}0.311 \mathrm{~W} \\
(0.335 \mathrm{~W}) \\
0.311 \mathrm{Q} \\
\end{array}$ & $\begin{array}{c}3190 \mathrm{~W} \\
(4330 \mathrm{~W}) \\
3190 \mathrm{Q} \\
\end{array}$ & $\begin{array}{c}0.317 \mathrm{~W} \\
(0.343 \mathrm{~W}) \\
0.328 \mathrm{Q}\end{array}$ & $\begin{array}{c}3370 \mathrm{~W} \\
(4740 \mathrm{~W}) \\
3510 \mathrm{Q} \\
\end{array}$ & $\begin{array}{c}0.322 \mathrm{~W} \\
(0354 \mathrm{~W}) \\
0.291 \mathrm{Q}\end{array}$ & $\begin{array}{c}3540 \mathrm{~W} \\
(5210 \mathrm{~W}) \\
3400 \mathrm{Q}\end{array}$ \\
\hline \multirow{2}{*}{$\begin{array}{l}\text { 1-phase 1-cycle Zero-crossing } \\
\text { (updated every half cycle) }\end{array}$} & & & 1 & $3 / 4^{*}$ & $\begin{array}{c}0.238 \mathrm{~W} \\
(0.252 \mathrm{~W}) \\
0.269 \mathrm{Q} \\
(0.247 \mathrm{Q})\end{array}$ & $-\mathrm{B}=\mathrm{C}$ & $\begin{array}{c}0.253 \mathrm{~W} \\
(0.253 \mathrm{~W}) \\
0.329 \mathrm{Q} \\
(0.215 \mathrm{Q}) \\
\end{array}$ & & $\begin{array}{c}0.261 \\
(0.252 \mathrm{~W}) \\
0.268 \mathrm{Q} \\
(0.361 \mathrm{Q})\end{array}$ & \\
\hline & & & $1 \frac{1}{2}$ & $1^{*}$ & 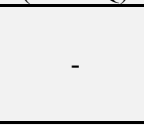 & $\begin{array}{c}33.8 \mathrm{~W} \\
(35.7 \mathrm{~W}) \\
33.8 \mathrm{Q} \\
(34.9 \mathrm{Q}) \\
\end{array}$ & & $\begin{array}{c}35.9 \mathrm{~W} \\
(35.8 \mathrm{~W}) \\
46.7 \mathrm{Q} \\
(30.4 \mathrm{Q}) \\
\end{array}$ & & $\begin{array}{c}36.4 \mathrm{~W} \\
(35.8 \mathrm{~W}) \\
38.1 \mathrm{Q} \\
(51.1 \mathrm{Q})\end{array}$ \\
\hline \multirow{2}{*}{$\begin{array}{l}\text { 1-phase 1-cycle Zero-crossing } \\
\text { (updated every full cycle) }\end{array}$} & & & 1 & $1^{*}$ & $\begin{array}{c}0.224 \mathrm{~W} \\
(0.252 \mathrm{~W}) \\
0.270 \mathrm{Q} \\
(0.247 \mathrm{Q}) \\
\end{array}$ & $x^{2}$ & $\begin{array}{c}0.260 \mathrm{~W} \\
(0.253 \mathrm{~W}) \\
0.269 \mathrm{Q} \\
(0.215 \mathrm{Q}) \\
\end{array}$ & & $\begin{array}{c}0.285 \mathrm{~W} \\
(0.252 \mathrm{~W}) \\
0.380 \mathrm{Q} \\
(0.361 \mathrm{Q}) \\
\end{array}$ & \\
\hline & & & 2 & $1 \frac{1}{2}{ }^{*}$ & 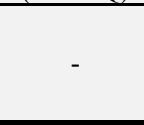 & $\begin{array}{c}19.0 \mathrm{~W} \\
(17.8 \mathrm{~W}) \\
26.2 \mathrm{Q} \\
(17.5 \mathrm{Q})\end{array}$ & & $\begin{array}{c}22.3 \mathrm{~W} \\
(17.9 \mathrm{~W}) \\
23.3 \mathrm{Q} \\
(15.2 \mathrm{Q})\end{array}$ & & $\begin{array}{c}24.4 \mathrm{~W} \\
(17.9 \mathrm{~W}) \\
38.1 \mathrm{Q} \\
(25.6 \mathrm{Q})\end{array}$ \\
\hline 3 phase Heterodyne & 1 & $\{1\}$ & 1 & $1 / 2$ & $\begin{array}{c}0.180 \mathrm{~W} \\
(0.193 \mathrm{~W}) \\
0.181 \mathrm{Q} \\
\end{array}$ & $\begin{array}{c}1860 \mathrm{~W} \\
(2500 \mathrm{~W}) \\
1870 \mathrm{Q}\end{array}$ & $\begin{array}{c}0.182 \mathrm{~W} \\
(0.199 \mathrm{~W}) \\
0.177 \mathrm{Q}\end{array}$ & $\begin{array}{c}1930 \mathrm{~W} \\
(2740 \mathrm{~W}) \\
1950 \mathrm{Q}\end{array}$ & $\begin{array}{c}0.186 \mathrm{~W} \\
(0.204 \mathrm{~W}) \\
0.174 \mathrm{Q}\end{array}$ & $\begin{array}{c}2050 \mathrm{~W} \\
(3010 \mathrm{~W}) \\
2170 \mathrm{Q}\end{array}$ \\
\hline 3 phase Clarke/Park transform & 1 & $\{1\}$ & 1 & $1 / 2$ & $\begin{array}{c}0.180 \mathrm{~W} \\
(0.193 \mathrm{~W}) \\
0.181 \mathrm{Q} \\
\end{array}$ & $\begin{array}{c}1860 \mathrm{~W} \\
(2500 \mathrm{~W}) \\
1870 \mathrm{Q} \\
\end{array}$ & $\begin{array}{c}0.182 \mathrm{~W} \\
(0.199 \mathrm{~W}) \\
0.177 \mathrm{Q}\end{array}$ & $\begin{array}{c}1930 \mathrm{~W} \\
(2740 \mathrm{~W}) \\
1950 \mathrm{Q} \\
\end{array}$ & $\begin{array}{c}0.186 \mathrm{~W} \\
(0.204 \mathrm{~W}) \\
0.174 \mathrm{Q}\end{array}$ & $\begin{array}{c}2050 \mathrm{~W} \\
(3010 \mathrm{~W}) \\
2170 \mathrm{Q} \\
\end{array}$ \\
\hline \multirow{2}{*}{$\begin{array}{l}\text { 3-phase 1-cycle Zero-crossing } \\
\text { (updated every half cycle) }\end{array}$} & & & 1 & $3 / 4^{*}$ & $\begin{array}{c}0.145 \mathrm{~W} \\
(0.146 \mathrm{~W}) \\
0.136 \mathrm{Q} \\
(0.143 \mathrm{Q}) \\
\end{array}$ & - & $\begin{array}{c}0.145 \mathrm{~W} \\
(0.146 \mathrm{~W}) \\
0.129 \mathrm{Q} \\
(0.124 \mathrm{Q}) \\
\end{array}$ & & $\begin{array}{c}0.147 \mathrm{~W} \\
(0.146 \mathrm{~W}) \\
0.090 \mathrm{Q} \\
(0.208 \mathrm{Q}) \\
\end{array}$ & \\
\hline & & & $11 / 2$ & $1 *$ & - & $\begin{array}{c}20.4 \mathrm{~W} \\
(20.6 \mathrm{~W}) \\
20.4 \mathrm{Q} \\
(20.1 \mathrm{Q})\end{array}$ & & $\begin{array}{c}20.3 \mathrm{~W} \\
(20.7 \mathrm{~W}) \\
17.6 \mathrm{Q} \\
(17.6 \mathrm{Q})\end{array}$ & & $\begin{array}{c}20.7 \mathrm{~W} \\
(20.7 \mathrm{~W}) \\
12.7 \mathrm{Q} \\
(29.6 \mathrm{Q})\end{array}$ \\
\hline \multirow{2}{*}{$\begin{array}{l}\text { 3-phase 1-cycle Zero-crossing } \\
\text { (updated every full cycle) }\end{array}$} & & & 1 & $1^{*}$ & $\begin{array}{c}0.137 \mathrm{~W} \\
(0.146 \mathrm{~W}) \\
0.126 \mathrm{Q} \\
(0.143 \mathrm{Q}) \\
\end{array}$ & - & $\begin{array}{c}0.149 \mathrm{~W} \\
(0.146 \mathrm{~W}) \\
0.141 \mathrm{Q} \\
(0.124 \mathrm{Q}) \\
\end{array}$ & & $\begin{array}{c}0.163 \mathrm{~W} \\
(0.146 \mathrm{~W}) \\
0.127 \mathrm{Q} \\
(0.208 \mathrm{Q}) \\
\end{array}$ & \\
\hline & & & 2 & $1 \frac{1}{2}{ }^{*}$ & - & $\begin{array}{c}11.7 \mathrm{~W} \\
(10.3 \mathrm{~W}) \\
12.1 \mathrm{Q} \\
(10.1 \mathrm{Q})\end{array}$ & & $\begin{array}{c}12.8 \mathrm{~W} \\
(10.3 \mathrm{~W}) \\
11.2 \mathrm{Q} \\
(8.8 \mathrm{Q})\end{array}$ & & $\begin{array}{c}14.1 \mathrm{~W} \\
(10.3 \mathrm{~W}) \\
12.7 \mathrm{Q} \\
(14.8 \mathrm{Q})\end{array}$ \\
\hline $\begin{array}{l}\text { 3-phase Heterodyne or } \\
\text { Clarke/Park }\end{array}$ & 1 & $\{2\}$ & 2 & 1 & $\begin{array}{c}0.0919 \mathrm{~W} \\
(0.0993 \mathrm{~W}) \\
0.907 \mathrm{Q}\end{array}$ & $\begin{array}{c}983 \mathrm{~W} \\
(1380 \mathrm{~W}) \\
969 \mathrm{Q}\end{array}$ & $\begin{array}{c}0.0935 \mathrm{~W} \\
(0.1019 \mathrm{~W}) \\
0.0921 \mathrm{Q}\end{array}$ & $\begin{array}{c}1020 \mathrm{~W} \\
(1500 \mathrm{~W}) \\
1010 \mathrm{Q}\end{array}$ & $\begin{array}{c}0.0896 \mathrm{~W} \\
(0.0953 \mathrm{~W}) \\
0.0000 \mathrm{Q}\end{array}$ & $\begin{array}{c}899 \mathrm{~W} \\
(1180 \mathrm{~W}) \\
0.00 \mathrm{Q}\end{array}$ \\
\hline $\begin{array}{l}\text { 3-phase Heterodyne or } \\
\text { Clarke/Park }\end{array}$ & 2 & $\{1,1\}$ & 2 & 1 & $\begin{array}{c}0.0166 \mathrm{~W} \\
(0.0179 \mathrm{~W}) \\
0.0165 \mathrm{Q}\end{array}$ & $\begin{array}{c}13.4 \mathrm{~W} \\
(14.6 \mathrm{~W}) \\
13.3 \mathrm{Q} \\
\end{array}$ & $\begin{array}{c}0.0170 \mathrm{~W} \\
(0.0179 \mathrm{~W}) \\
0.0211 \mathrm{Q}\end{array}$ & $\begin{array}{c}13.4 \mathrm{~W} \\
(14.9 \mathrm{~W}) \\
12.8 \mathrm{Q} \\
\end{array}$ & $\begin{array}{c}0.0182 \mathrm{~W} \\
(0.0179 \mathrm{~W}) \\
0.0082 \mathrm{Q}\end{array}$ & $\begin{array}{c}13.7 \mathrm{~W} \\
(15.2 \mathrm{~W}) \\
14.0 \mathrm{Q} \\
\end{array}$ \\
\hline \multirow{2}{*}{$\begin{array}{l}\text { 3-phase 2-cycle Zero-crossing } \\
\text { (updated every half cycle) }\end{array}$} & & & 2 & $1 \frac{1 / 4}{*}$ & $\begin{array}{c}0.0736 \mathrm{~W} \\
(0.0729 \mathrm{~W}) \\
0.0423 \mathrm{Q} \\
(0.0658 \mathrm{Q}) \\
\end{array}$ & - & $\begin{array}{c}0.0709 \mathrm{~W} \\
(0.0728 \mathrm{~W}) \\
0.0859 \mathrm{Q} \\
(0.0807 \mathrm{Q}) \\
\end{array}$ & & $\begin{array}{c}0.0736 \mathrm{~W} \\
(0.0730 \mathrm{~W}) \\
0.0000 \mathrm{Q} \\
(0.0329 \mathrm{Q}) \\
\end{array}$ & \\
\hline & & & $2 \frac{1}{2}$ & $1 \frac{1 / 2}{}{ }^{*}$ & - & $\begin{array}{c}10.4 \mathrm{~W} \\
(10.3 \mathrm{~W}) \\
6.56 \mathrm{Q} \\
(9.31 \mathrm{Q}) \\
\end{array}$ & & $\begin{array}{c}10.1 \mathrm{~W} \\
(10.3 \mathrm{~W}) \\
6.08 \mathrm{Q} \\
(11.4 \mathrm{Q}) \\
\end{array}$ & & $\begin{array}{c}10.3 \mathrm{~W} \\
(10.4 \mathrm{~W}) \\
0.00 \mathrm{Q} \\
(4.67 \mathrm{Q})\end{array}$ \\
\hline \multirow{2}{*}{$\begin{array}{l}\text { 3-phase 2-cycle Zero-crossing } \\
\text { (updated every full cycle) }\end{array}$} & & & 2 & $1 \frac{1}{2}{ }^{*}$ & $\begin{array}{c}0.0740 \mathrm{~W} \\
(0.0729 \mathrm{~W}) \\
0.0364 \mathrm{Q} \\
(0.0658 \mathrm{Q}) \\
\end{array}$ & $(8,0)$ & $\begin{array}{c}0.0773 \mathrm{~W} \\
(0.0728 \mathrm{~W}) \\
0.0859 \mathrm{Q} \\
(0.0807 \mathrm{Q})\end{array}$ & & $\begin{array}{c}0.0817 \mathrm{~W} \\
(0.0730 \mathrm{~W}) \\
0.0000 \mathrm{Q} \\
(0.0329 \mathrm{Q})\end{array}$ & \\
\hline & & & 3 & $2^{*}$ & - & $\begin{array}{c}5.27 \mathrm{~W} \\
(5.16 \mathrm{~W}) \\
2.93 \mathrm{Q} \\
(4.65 \mathrm{Q})\end{array}$ & & $\begin{array}{c}5.37 \mathrm{~W} \\
(5.15 \mathrm{~W}) \\
6.08 \mathrm{Q} \\
(5.71 \mathrm{Q})\end{array}$ & & $\begin{array}{c}5.73 \mathrm{~W} \\
(5.18 \mathrm{~W}) \\
0.00 \mathrm{Q} \\
(2.34 \mathrm{Q})\end{array}$ \\
\hline
\end{tabular}

